\title{
TERT promoter hypermethylation is associated with poor prognosis in adrenocortical carcinoma
}

\author{
FREDRIKA SVAHN $^{1}$, JOHAN O. PAULSSON ${ }^{1}$, ADAM STENMAN ${ }^{1}$, OMID FOTOUHI ${ }^{1}$, \\ NINNI MU ${ }^{1}$, TIMOTHY D. MURTHA ${ }^{2}$, REJU KORAH $^{2}$, TOBIAS CARLING $^{2}$, MARTIN BÄCKDAHL $^{3}$, \\ NA WANG $^{1}$, C. CHRISTOFER JUHLIN ${ }^{1}$ and CATHARINA LARSSON ${ }^{1}$ \\ ${ }^{1}$ Department of Oncology-Pathology, Karolinska Institutet, Karolinska University Hospital, Cancer Center Karolinska, \\ SE-17176 Stockholm, Sweden; ${ }^{2}$ Department of Surgery, Yale School of Medicine, Yale Endocrine Neoplasia Laboratory, \\ Yale School of Medicine, New Haven, CT 06520, USA; ${ }^{3}$ Department of Molecular Medicine and Surgery, \\ Karolinska Institutet, Karolinska University Hospital, SE-17176 Stockholm, Sweden
}

Received January 10, 2018; Accepted May 11, 2018

DOI: $10.3892 / \mathrm{ijmm} .2018 .3735$

\begin{abstract}
Telomere maintenance, most commonly achieved by telomerase activation through induction of the telomerase reverse transcriptase (TERT) gene, is required for cell immortalization, a hallmark of cancer. Adrenocortical carcinoma (ACC) is an endocrine tumor for which TERT promoter mutations and telomerase activation have been reported. The present study assessed alterations of the TERT gene locus and telomere length in relation to clinical characteristics in ACC. In total, 38 cases of ACC with known TERT promoter mutational status were included. TERT promoter methylation densities were assessed by pyrosequencing, and TERT copy numbers and telomere length were determined by quantitative polymerase chain reaction analysis, followed by comparison of the mRNA expression of TERT and clinical parameters. The ACC tissue samples showed increased TERT copy numbers, compared with normal adrenal tissue (NAT) samples ( $\mathrm{P}=0.001)$. Mutually exclusive TERT copy number gains or promoter mutation were present in $70 \%$ of the ACC samples. The ACC tissues exhibited higher levels of $\mathrm{CpG}$ promoter methylation of all eight CpG sites investigated within the -578 to -541 bp (Region A), compared with the NATs $(\mathrm{P}=0.001)$. High methylation density at this region was associated with metastatic disease and/or relapse, poor survival rates and higher European Network for the Study of Adrenal Tumor stage $(\mathrm{P}<0.05)$. The mRNA expression of TERT was inversely correlated with methylation
\end{abstract}

Correspondence to: Dr Fredrika Svahn or Dr Adam Stenman, Department of Oncology-Pathology, Karolinska Institutet, Karolinska University Hospital, Cancer Center Karolinska, Solna R8:04, SE-17176 Stockholm, Sweden

E-mail: fredrika.svahn@ki.se

E-mail: adam.stenman@ki.se

Key words: telomerase reverse transcriptase, methylation, copy number, adrenocortical carcinoma, telomere length density at -162 to -100 bp (Region B). Correlation was observed between relative telomere length and the gene expression of $T E R T$. It was concluded that epigenetic alterations of the TERT promoter are frequent and associated with advanced disease and poorer clinical outcome in ACC.

\section{Introduction}

Telomerase reverse transcriptase (TERT) is a subunit of the telomerase enzyme complex, which can increase telomere length by adding TTAGGG repeats $(1,2)$. As telomeres become shorter for each cell division, this addition of nucleotides increases the possible number of cell divisions. In cancer, telomerase activation is frequently observed. TERT promoter mutations have been associated with more aggressive disease and therefore suggested as a prognostic marker (2). Shorter telomeric repeat sequences are commonly observed in tumor tissues, further supporting the telomere involvement (3).

Adrenocortical carcinoma (ACC) is a rare endocrine tumor with a generally poor prognosis. The majority of patients present with metastatic or inoperable disease, and despite initial tumor-free resection margin surgery, a majority of patients relapse or develop progressive disease (4). Mitotane (Lysodren) is used as a primary therapy and adjuvant treatment (5); however, it has serious and ill-tolerated side effects (6). The majority of cases of ACC arise in a sporadic setting and exhibit frequent somatic mutations in multiple genes, including the driver genes tumor protein 53 (TP53), catenin $\beta 1$, zinc and ring dinger 3 , and protein kinase cAMP-dependent type I regulatory subunit $\alpha$ (7-9).

The upregulation of TERT and telomerase activity have previously been reported in ACC $(10,11)$. One genetic mode for the upregulation of TERT stems from the recurrent TERT promoter mutations $\mathrm{C} 228 \mathrm{~T}$ and $\mathrm{C} 250 \mathrm{~T}$, which were first described in patients with melanoma (12). Such mutations have been found in ACC, providing a possible explanation for the observed expression of TERT in subsets of this tumor type. However, as the expression of TERT was also found in TERT wild-type cases, additional genetic or epigenetic mechanisms 
are expected to be involved (10). In ACC, TERT copy number gain has been found as a recurrent genetic event and mutually exclusive from established driver gene mutations (7). However, the occurrence and impact on disease presentation of epigenetic modifications, including TERT promoter methylation, remains to be elucidated in ACC. Studies of other cancer types have identified regions of high methylation in association with poor patient outcome, including at positions -578 to $-541 \mathrm{bp}$ in medullary thyroid carcinoma (Region A, Fig. 1A) (13), and a region located -162 to $-100 \mathrm{bp}$ infront of the transcriptional start site (Region B, Fig. 1A) in which low methylation was shown to be required for the expression of TERT in vitro (14).

In the present study, TERT promoter methylation density, copy number and telomere length were assessed in relation to clinical characteristics in ACC samples, which were also characterized for TERT promoter mutations and TERT gene expression.

\section{Patients and methods}

Tumor samples and references. Samples were collected in accordance with the ethical permits obtained from the Ethical Committee of Karolinska Institutet (Stockholm, Sweden) and from the International Review Board at Yale University (New Haven, CT, USA). Tissue material was collected with informed consent obtained from patients prior to surgery.

In total, 38 tumor samples were used in the experiments. Of the tumors, 27 (ACC-1-27) were collected and diagnosed at the Karolinska University Hospital (Stockholm, Sweden) between July 1986 and July 2009. In this cohort, 16 were female and 11 were male, with an age range of 28 to 84 years old (mean age, 59.4 years). Six patients had metastases at the time of surgery. Samples were snap frozen in liquid nitrogen in conjunction with postoperative pathological grossing. All of these tissues were histopathologically confirmed and samples with $<50 \%$ tumor cells were excluded from the study. The remaining 11 samples (ACC-Y1-Y11) were collected between August 2003 and July 2015 at Yale University. In this cohort, 8 patients were female and 3 were male, with an age range of 18 to 69 years (mean age, 52.2 years) at surgery. Two of the patients had metastasis at the time of surgery. Genomic DNA was extracted from formalin-fixated paraffin-embedded samples.

The following adrenal references were used for the investigation of methylation levels and the devaluation of relative telomere length: Eight histologically confirmed normal adrenal tissue samples (comprising a majority of adrenocortical cells), from renal or adrenal surgical specimens; two commercially available adrenal medullary DNA (Clinomics, Watervliet, NY, USA); (NAT, $\mathrm{n}=10$ ); and adrenocortical hyperplasia $(\mathrm{ACH})$ tissue samples ( $\mathrm{n}=8$, only for relative telomere length). For the copy number analysis, 14 histologically confirmed NAT samples were used in addition to the two commercially available adrenal medullary DNA (NAT=16).

The tumors were diagnosed according to the criteria of the 2004 World Health Organization classification (15). The ACC cases were clinically characterized for sex, age at surgery, tumor size (largest diameter), tumor weight, MIB1 proliferation index (based on Ki-67 immunohistochemistry) European Network for the Study of Adrenal Tumors (ENSAT) staging score, metastasis, and outcome. Some of the clinical information has been previously published $(7,10)$.

TERT promoter mutation status. Of the tumors, 27 were previously screened for the TERT promoter hot spot mutations C228T and C250T and investigated for quantification of the mRNA expression of TERT (ACC-1-27; Table I) (10). The other 11 samples (ACC-Y1-Y11) were investigated for TERT promoter mutation status and the analysis was successfully performed for eight of these samples. The mutation status was determined using Sanger sequencing, applying a previously described methodology (10), which revealed that one of the eight samples showed the $\mathrm{C} 228 \mathrm{~T}$ mutation and the remaining seven samples were wild-type (Table I).

DNA copy number. Genomic DNA was extracted from tumour tissues using a DNAeasy Blood and Tissue DNA isolation kit (Qiagen AB, Sollentuna, Sweden). A total of 27 ACC samples was successfully analyzed for TERT copy numbers. DNA at $5 \mathrm{ng} / \mu \mathrm{l}$ was analyzed (with five repeats) with the TaqMan assays (Applied Biosystems; Thermo Fisher Scientific, Inc., Waltham, MA, USA) Hs 01237576_CN for TERT, with Hs 4403326_C for the RNaseP gene used as an endogenous control. Following normalization to RNaseP, data were calibrated to NAT DNA. The data were exported to CopyCaller v2.0 (Applied Biosystems; Thermo Fisher Scientific, Inc.) and the copy numbers were estimated.

Pyrosequencing. The TERT promoter regions investigated for methylation density are shown in Fig. 1A. CpG methylation was quantified at eight sites within Region A located between -578 and -541 bp, and five sites within Region B between -162 and -100 bp. DNA from a total of 35 ACC samples was successfully analyzed for Region A, and from 37 ACC samples for Region B.

The methodology for pyrosequencing was as previously described (13), and the primers used are detailed in Table II. For Region B, the primers were designed in-house with PyroMark Assay Design 2.0 (Qiagen AB). The majority of samples were fresh frozen following surgery and a minority of the samples was paraffin-embedded. Previous studies have demonstrated the validity of both materials in sequencing experiments (16). Bisulphite treatment was performed with the EpiTect bisulphite kit (Qiagen AB). Polymerase chain reaction (PCR) amplification of the bisulphite converted DNA was performed with the Qiagen PCR kit (Qiagen AB), including 20 ng DNA, 12.5 $\mu$ l PyroMark PCR Master mix, $2.5 \mu 1$ Coral load, $7 \mu \mathrm{l} \mathrm{H}_{2} \mathrm{O}, 1 \mu \mathrm{l} \mathrm{MgCl}_{2}$ and $0.5 \mu \mathrm{l}$ primers $10 \mu \mathrm{M}$ for Region A (forward, 5'-GGGTTTGTGTTAAGGAGTTTA AGT-3' and reverse, 5'-AAACCCAAAACTACC TCCA-3') and $5 \mu \mathrm{M}$ for Region B (forward, 5'-GGTGGTAGGGGTTAG GGTTTTTTA-3' and reverse, 5'-TACCCCTTCACCTTCCAA CTC-3'). PCR amplifications included 45 cycles of $30 \mathrm{sec}$ at $94^{\circ} \mathrm{C}, 30 \mathrm{sec}$ at $58^{\circ} \mathrm{C}$ for Region $\mathrm{A}$ and at $56^{\circ} \mathrm{C}$ for Region $\mathrm{B}$, and $30 \mathrm{sec}$ at $72^{\circ} \mathrm{C}$. The pyrosequencing reactions were run in a PyroMark Q24 system and the data were analyzed with PyroMark Q24 2.0.7 software (Qiagen AB). For each sample, a methylation index (MetI) was calculated as the mean methylation density of the investigated $\mathrm{CpG}$ for Region $\mathrm{A}$ and Region B, respectively. For survival analysis, the cut-off for 
A

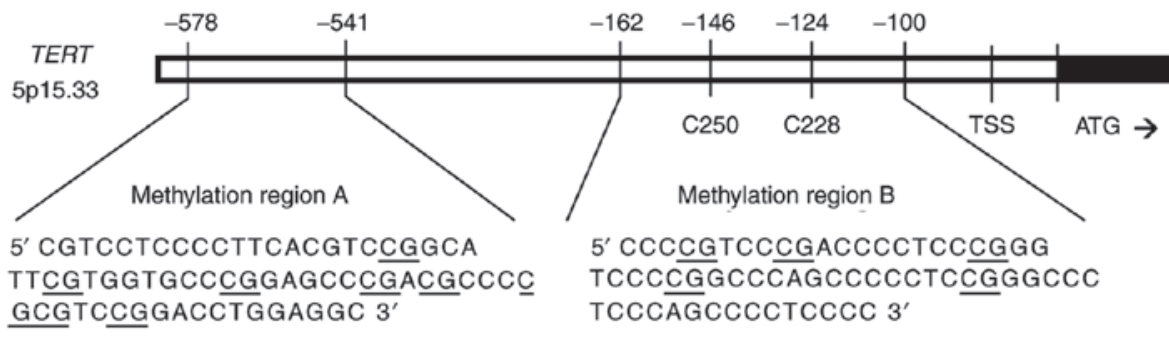

B
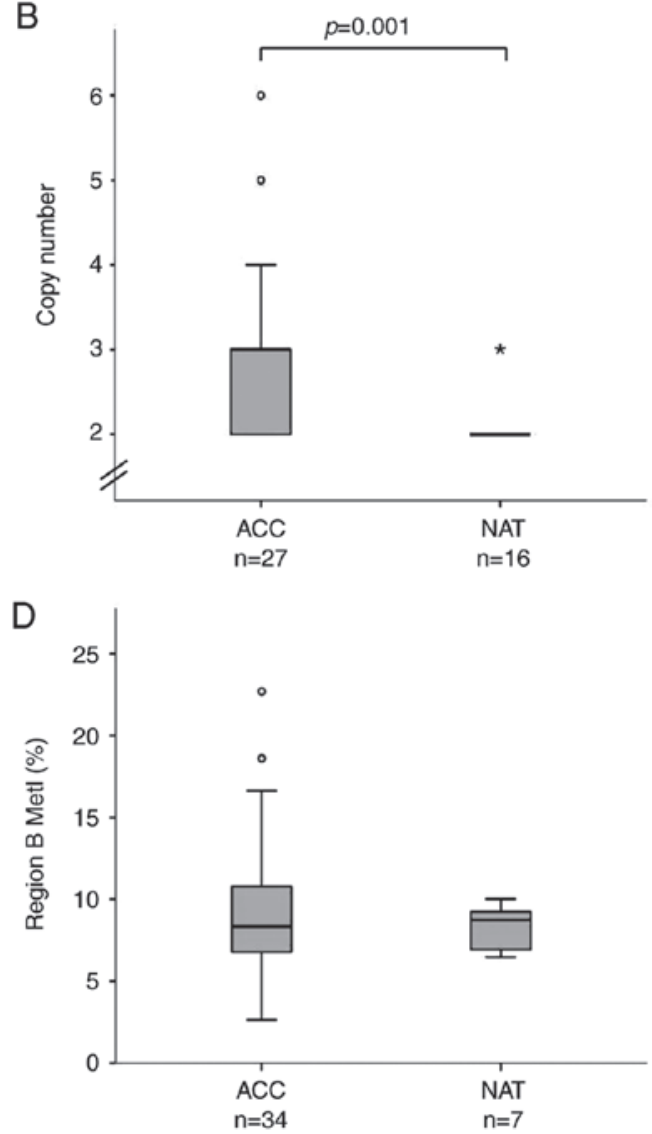

C
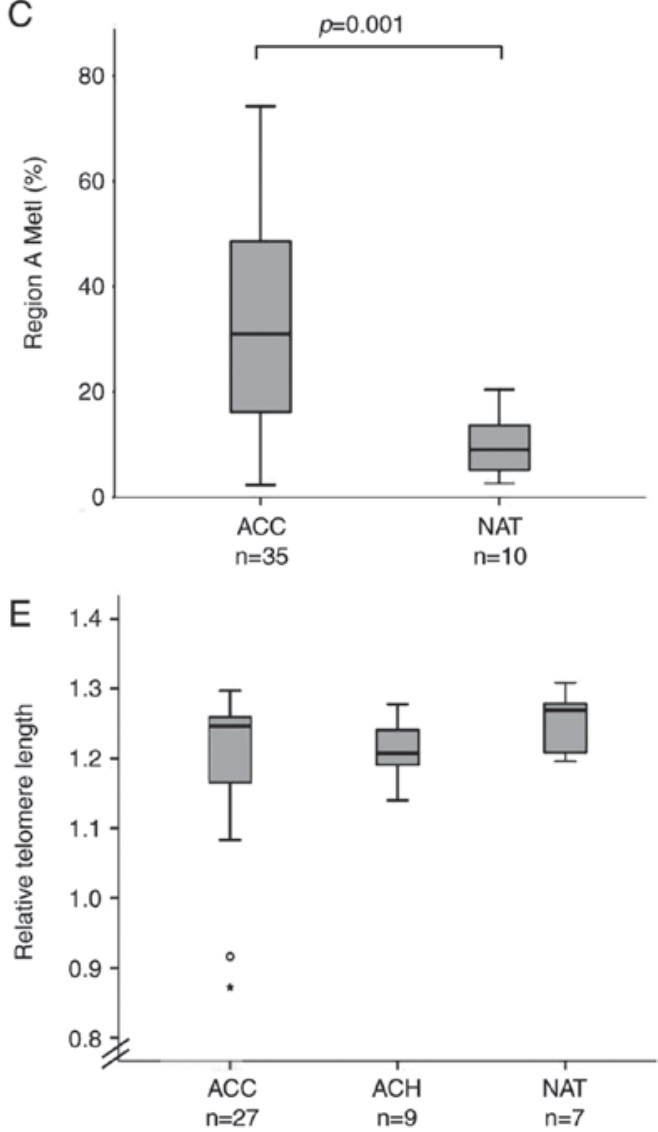

Figure 1. TERT promoter methylation sites and boxplot presentation of TERT promoter methylation densities, TERT copy number analysis and telomere length investigation. (A) Schematic illustration of the TERT promoter region in chromosomal region 5p15.33. Investigated CpG sites are underlined. The common mutation sites C250 and C228 are indicated. ATG represents the start of translation, and TSS the transcription start site. (B) TERT copy numbers for ACC tissues and NAT samples. (C) MetI of Region A for ACC and NAT samples. (D) MetI of Region B for ACC and NAT samples. (E) Relative telomere lengths in ACC, ACH and NAT samples. Boxes correspond to interquartiles, bars to non-outlier ranges, and bold lines to medians. Outliers are indicated by circles and extreme values by stars. A broken vertical axis is used for graphs B and E. TERT, telomerase reverse transcriptase; ACC, adrenocortical carcinoma; NAT, normal adrenal tissue; $\mathrm{ACH}$, adrenocortical hyperplasia; MetI, methylation index.

high and low methylation in Region A was set at $20.4 \%$ based on the highest methylation level for NAT.

Telomere length. The relative telomere length was determined for the 27 ACC samples using a previously described methodology $(17,18)$ and the primers are detailed in Table II. DNA at $10 \mathrm{ng} / \mu \mathrm{l}$ was used to determine the mean relative telomere length of the tumors using quantitative (q)PCR amplification of telomere $(T E L)$ and $\beta$-globin $(H B G)$, the latter serving as an internal control. The following was included in the experiment: $5 \mu 1$ SYBR ${ }^{\circledR}$ Green Real-Time PCR Master Mix (Applied Biosystems; Thermo Fisher Scientific Inc.), $0.2 \mu 1$ primers (TEL forward, 5'-CGGTTTGTTTGGGTTTGGGTTTGG GTTTGGGTTTGGGTT-3' and reverse, 5'-GGCTTGCCT TACCCTTACCCT TACCC-TTACCCTTACCCT-3'; HBG forward, 5'-TGTGCTGGCCCATCACTTTG-3' and reverse, 5'-ACCAGCCA-CCACTTTCTGATAGG-3') in $10 \mu \mathrm{M}, 2.6 \mu \mathrm{l}$ $\mathrm{H}_{2} \mathrm{O}$ and 20 ng DNA. The thermocycling conditions were as follows: $50^{\circ} \mathrm{C}$ for $2 \mathrm{~min}, 95^{\circ} \mathrm{C}$ for $2 \mathrm{~min}, 40$ cycles of $95^{\circ} \mathrm{C}$ for $15 \mathrm{sec}$ and $60^{\circ} \mathrm{C}$ for $60 \mathrm{sec}$. All samples were run in triplicate. The data was analyzed in Excel 2010 (Microsoft Corporation, Redmond, WA, USA), whereby the mean Cq value of each sample was used in the calculations. Outliers were manually omitted. The ratio of TEL compared to $H B G$ was used to estimate the relative telomere length and was used for comparison between samples.

Statistical analyses, graphs and figures. Statistical analyses was performed and figures made using IBM SPSS 23 (IBM SPSS, Armonk, NY, USA). The results of MetI at 
Table I. TERT genetic and epigenetic data and telomere length in 38 cases of ACC.

\begin{tabular}{|c|c|c|c|c|c|c|c|}
\hline \multirow[b]{2}{*}{ Case } & \multirow[b]{2}{*}{ Cohort } & \multirow{2}{*}{$\begin{array}{c}T E R T \\
\text { copy no. }\end{array}$} & \multirow{2}{*}{$\begin{array}{c}\text { TERT } \\
\text { mutation }^{\mathrm{a}}\end{array}$} & \multicolumn{2}{|c|}{$\begin{array}{l}\text { TERT promoter } \\
\text { MetI \% (range) }\end{array}$} & \multirow{2}{*}{$\begin{array}{c}\text { TERT } \\
\text { mRNA }^{\mathrm{a}}\end{array}$} & \multirow{2}{*}{$\begin{array}{l}\text { Relative } \\
\text { telomere length }\end{array}$} \\
\hline & & & & Region A & Region B & & \\
\hline ACC-1 & Karolinska & 2 & No & $25(5-40)$ & $7(5-10)$ & 0.000389 & 0.92 \\
\hline ACC-2 & Karolinska & 2 & No & $31(7-49)$ & $19(7-52)$ & 0 & 0.87 \\
\hline ACC-3 & Karolinska & 3 & No & $50(40-60)$ & $8(5-13)$ & 0.049385 & 1.26 \\
\hline ACC-4 & Karolinska & 2 & No & $20(10-27)$ & $6(4-9)$ & 0.007639 & 1.21 \\
\hline ACC-5 & Karolinska & 3 & No & $8(3-15)$ & - & 0.017604 & 1.27 \\
\hline ACC-6 & Karolinska & 2 & No & $3(1-4)$ & $6(3-10)$ & 0.000408 & 1.17 \\
\hline ACC-7 & Karolinska & 2 & $\mathrm{C} 228 \mathrm{~T}$ & $41(9-63)$ & - & 0.001654 & 1.20 \\
\hline ACC-8 & Karolinska & 3 & No & $37(30-41)$ & $7(3-11)$ & 1.349172 & 1.25 \\
\hline ACC-9 & Karolinska & 5 & No & $46(24-71)$ & $10(5-17)$ & 0.000217 & 1.15 \\
\hline ACC-10 & Karolinska & 6 & No & $34(8-71)$ & $8(5-11)$ & 0.029137 & 1.23 \\
\hline ACC-11 & Karolinska & 3 & No & $8(3-14)$ & $8(3-15)$ & 0.269476 & 1.16 \\
\hline ACC-12 & Karolinska & 2 & $\mathrm{C} 228 \mathrm{~T}$ & $12(6-21)$ & $8(5-13)$ & 0.016867 & 1.26 \\
\hline ACC-13 & Karolinska & 2 & No & $45(15-60)$ & $11(7-18)$ & 0.000207 & 1.23 \\
\hline ACC-14 & Karolinska & 4 & No & $46(19-70)$ & $11(5-24)$ & 0.000997 & 1.26 \\
\hline ACC-15 & Karolinska & 3 & No & $57(9-90)$ & $9(5-14)$ & 0.000196 & 1.08 \\
\hline ACC-16 & Karolinska & 4 & No & $49(29-62)$ & $6(4-10)$ & 0.00856 & 1.25 \\
\hline ACC-17 & Karolinska & 3 & No & $31(12-51)$ & $6(3-10)$ & 0.00027 & 1.25 \\
\hline ACC-18 & Karolinska & 3 & No & $14(6-22)$ & $8(5-14)$ & 0.44975 & 1.30 \\
\hline ACC-19 & Karolinska & 2 & No & $48(12-88)$ & $6(3-8)$ & 0.292925 & 1.25 \\
\hline ACC-20 & Karolinska & 2 & No & $31(9-68)$ & $17(5-30)$ & 0 & 1.11 \\
\hline ACC-21 & Karolinska & 3 & No & $74(61-87)$ & $8(5-10)$ & 0.010663 & 1.28 \\
\hline ACC-22 & Karolinska & 3 & No & $60(40-85)$ & $10(4-14)$ & 0.019338 & 1.17 \\
\hline ACC-23 & Karolinska & 3 & No & $19(8-39)$ & $9(6-14)$ & 0.001294 & 1.25 \\
\hline ACC-24 & Karolinska & 2 & No & $13(2-25)$ & $9(7-11)$ & 0.000382 & 1.25 \\
\hline ACC-25 & Karolinska & 3 & No & $10(2-17)$ & $14(7-20)$ & 0 & 1.15 \\
\hline ACC-26 & Karolinska & 3 & No & $30(6-60)$ & $11(6-14)$ & 0.007517 & 1.27 \\
\hline ACC-27 & Karolinska & 2 & $\mathrm{C} 228 \mathrm{~T}$ & $28(10-54)$ & - & 0.006031 & 1.29 \\
\hline ACC-Y1 & Yale & - & No & $52(45-68)$ & $7(3-10)$ & - & - \\
\hline ACC-Y2 & Yale & - & No & $74(66-83)$ & $9(1-24)$ & - & - \\
\hline ACC-Y3 & Yale & - & No & $13(2-33)$ & $13(4-33)$ & - & - \\
\hline ACC-Y4 & Yale & - & No & $19(3-44)$ & $12(1-22)$ & - & - \\
\hline ACC-Y5 & Yale & - & No & $51(40-96)$ & $7(1-19)$ & - & - \\
\hline ACC-Y6 & Yale & - & $\mathrm{C} 228 \mathrm{~T}$ & $19(14-30)$ & $5(2-8)$ & - & - \\
\hline ACC-Y7 & Yale & - & No & 73 (40-89) & $23(8-73)$ & - & - \\
\hline ACC-Y8 & Yale & - & - & $2(1-4)$ & - & - & - \\
\hline ACC-Y9 & Yale & - & - & - & $5(1-19)$ & - & - \\
\hline ACC-Y10 & Yale & - & - & - & $10(1-43)$ & - & - \\
\hline ACC-Y11 & Yale & - & No & - & $3(2-4)$ & - & - \\
\hline
\end{tabular}

a Data for ACC 1-27 from Ref. (10). -, not determined or not informative; MetI, methylation index; TERT, telomerase reverse transcriptase; ACC, adrenocortical carcinoma.

Region A and B, TERT copy number and telomere length in the sample groups were compared with clinical parameters, TERT mutation status and expression. In addition, statistical analyses of TERT promoter methylation and clinical parameters, and survival rates were performed. The Mann-Whitney U test and Kruskal-Wallis test were used for comparison between groups. Correlations were assessed using Spearman's rank order. Disease-related and overall survival rates were analyzed using the Log-rank test and illustrated with Kaplan-Meier plots. $\mathrm{P}<0.05$ was considered to indicate a statistically significant difference. 
Table II. Primers used for methylation and telomere length analyses.

\begin{tabular}{lll}
\hline Gene primer & Primer direction & \multicolumn{1}{c}{ Primer sequence } \\
\hline $\begin{array}{l}\text { Promoter methylation region A } \\
\text { TERT }\end{array}$ & Forward & 5'-GGGTTTGTGTTAAGGAGTTTAAGT-3' \\
$T E R T$ & Reverse & 5'-AAACCCAAAACTACCTCCA-3' \\
$T E R T$ & Sequencing & 5'-CCAAAACTACCTCCAAAT-3' \\
Promoter methylation region B & & \\
$T E R T$ & Forward & 5'-GGTGGTAGGGGTTAGGGTTTTTTA-3' \\
$T E R T$ & Reverse & 5-TACCCCTTCACCTTCCAACTC-3' \\
$T E R T$ & Sequencing & 5'-GGGGTAGAGGAAAGGAA-3' \\
Relative telomere length & & \\
$T e l$ b & & \\
$T e l$ b & Forward & 5'-CGGTTTGTTTGGGTTTGGGTTTGGGTTTGGGTTTGGGTT-3' \\
$H B G 1$ & Reverse & 5'-GGCTTGCCTTACCCTTACCCTTACCC-TTACCCTTACCCT-3' \\
$H B G 2$ & Forward & 5'-TGTGCTGGCCCATCACTTTG-3' \\
\hline
\end{tabular}

Primers for telomere length are according to Ref (18). TERT, telomerase reverse transcriptase; Tel, telomere.

\section{Results}

Evaluation of the TERT gene locus. The TERT gene locus was investigated for methylation densities at Region A and Region B, in addition to DNA copy numbers and telomere length in a panel of ACC samples. The overall results from these investigations are shown in Fig. 1B-E and Table I. The acquired results were subsequently used in statistical comparisons with clinical data, in addition to data for TERT promoter mutations and the gene expression of TERT (Table III).

TERT copy number gain in ACC. The mean TERT copy number of 2.9 was observed for the ACCs (range 2-6, median 3) and of 2.1 for the NATs (Fig. 1B). The ACCs had a significantly higher copy number, compared with the NATs $(\mathrm{P}=0.001$, Mann-Whitney U test) (Figs. 1B and 2). In total, 19/27 cases (70\%) exhibited either copy number gain $(16 / 27,59 \%)$ or promoter mutation $(3 / 27,11 \%)$ (Fig. 2), without overlap. In 24/27 ACCs (89\%) TERT mRNA expression was present, however, no association was observed between the expression of TERT and copy number gain or promoter mutation.

Association of high methylation in Region A with poor outcome and advanced disease. The mean MetI in Region A was $33.5 \%$ in the ACCs (range 2.3-74.2\%, median 31.0\%) and $10.2 \%$ in the NATs (range 2.6-20.4\%, median 9.0\%). Similar patterns were observed with regard to the individual $\mathrm{CpGs}$ investigated (Fig. 2B).

The ACC group had a significantly higher MetI, compared with the NAT group ( $\mathrm{P}=0.001$, Mann-Whitney U test) (Figs. 1C and 2). No correlation was observed between Region A MetI and the mRNA expression levels of TERT.

Comparisons with clinical characteristics of the ACC samples revealed that higher MetIs for Region A were associated with metastases or relapse of disease $(\mathrm{P}<0.01$, Mann-Whitney U test), with poor patient outcome $(\mathrm{P}<0.05$, Mann-Whitney $U$ test) and higher ENSAT stage $(r=0.39$;
$\mathrm{P}<0.05$, Spearman's rank order correlation) (Table III; Fig. 3A and B). Patients with MetI $>20.4 \%$ had shorter disease-related survival and absolute survival rates $(\mathrm{P}<0.05$, Log-rank test), compared with the patients with MetI $<20.4 \%$ (Fig. 3C and D).

Association between methylation levels in Region $B$ and the mRNA expression of TERT in ACC. In Region B, a mean MetI of $9.3 \%$ was observed in the ACC group (range 2.6-22.7\%, median $8.3 \%$ ) and of $8.2 \%$ in the NAT group (range $6.5-10.0 \%$, median $8.8 \%$ ). Overall, no difference in MetI was observed between the ACC and NAT groups (Fig. 1D; Table I).

In the ACCs, MetI of Region B was inversely correlated with the mRNA expression levels of TERT $(\mathrm{P}<0.05, \mathrm{r}=-0.554$, Spearman's rank order correlation, Table III). All of the 24 ACC samples that exhibited mRNA expression of TERT had a MetI $<11.25 \%$, and the highest MetIs were noted in the three ACC samples without traceable expression of TERT (Fig. 2A and $\mathrm{B})$. The difference was particularly pronounced for $\mathrm{CpG}$ number 2 (Fig. 2B).

Correlation between relative telomere length and $m R N A$ expression of TERT in ACC. The relative telomere lengths were determined, which revealed a mean of 1.20 in the ACC group (range 0.9-1.3, median 1.25), of 1.21 in the $\mathrm{ACH}$ group (range 1.14-1.28, median 1.2) and of 1.25 (range 1.20-1.31, median 1.27) in the NAT group. No difference was observed between the ACC group and the NAT or ACH group (Fig. 1E). In the ACC group, the relative telomere length was positively correlated with the mRNA expression of TERT $(\mathrm{r}=0.57$, $\mathrm{P}<0.05$, Spearman's rank correlation, Table III). Furthermore, shorter telomere length was associated with poorer outcome $(\mathrm{P}<0.05$, Mann Whitney U test; Table III).

\section{Discussion}

Telomerase activity has been shown to be present in ACC (19). TERT promoter mutations, which may be one underlying 
Table III. Summary of statistical analyses.

\begin{tabular}{lcccr}
\hline Statistical test parameter & Copy number & Region A MetI & Region B MetI & Telomere le \\
\hline Mann-Whitney U test & & & & \\
TERT mutation & $\mathbf{0 . 0 4 4}$ & 0.285 & 0.213 & 0.247 \\
Outcome & 0.521 & $\mathbf{0 . 0 3 3}$ & 0.382 & $\mathbf{0 . 0 2 7}$ \\
Absolute outcome & 0.555 & 0.051 & 0.086 & $\mathbf{0 . 0 4 8}$ \\
Metastasis/relapse & 0.732 & $\mathbf{0 . 0 0 8}$ & 0.358 & 0.133 \\
Sex & 0.668 & 0.657 & 0.330 & 0.921 \\
Spearman's rank order correlation & & & & 0.573 \\
Tumor weight & 0.152 & 0.623 & 0.349 & 0.936 \\
Tumor size & 0.196 & 0.756 & 0.153 & 0.566 \\
ENSAT stage & 0.714 & $\mathbf{0 . 0 2 1}$ & 0.142 & 0.807 \\
Ki-67 index & 0.949 & 0.107 & 0.476 & $\mathbf{0 . 0 0 2}$ \\
TERT mRNA & 0.224 & 0.79 & $\mathbf{0 . 0 0 5}$ &
\end{tabular}

Statistically significant P-values $(\mathrm{P}<0.05)$ are indicated in bold. MetI, methylation index; ENSAT, European Network for the Study of Adrenal Tumors.
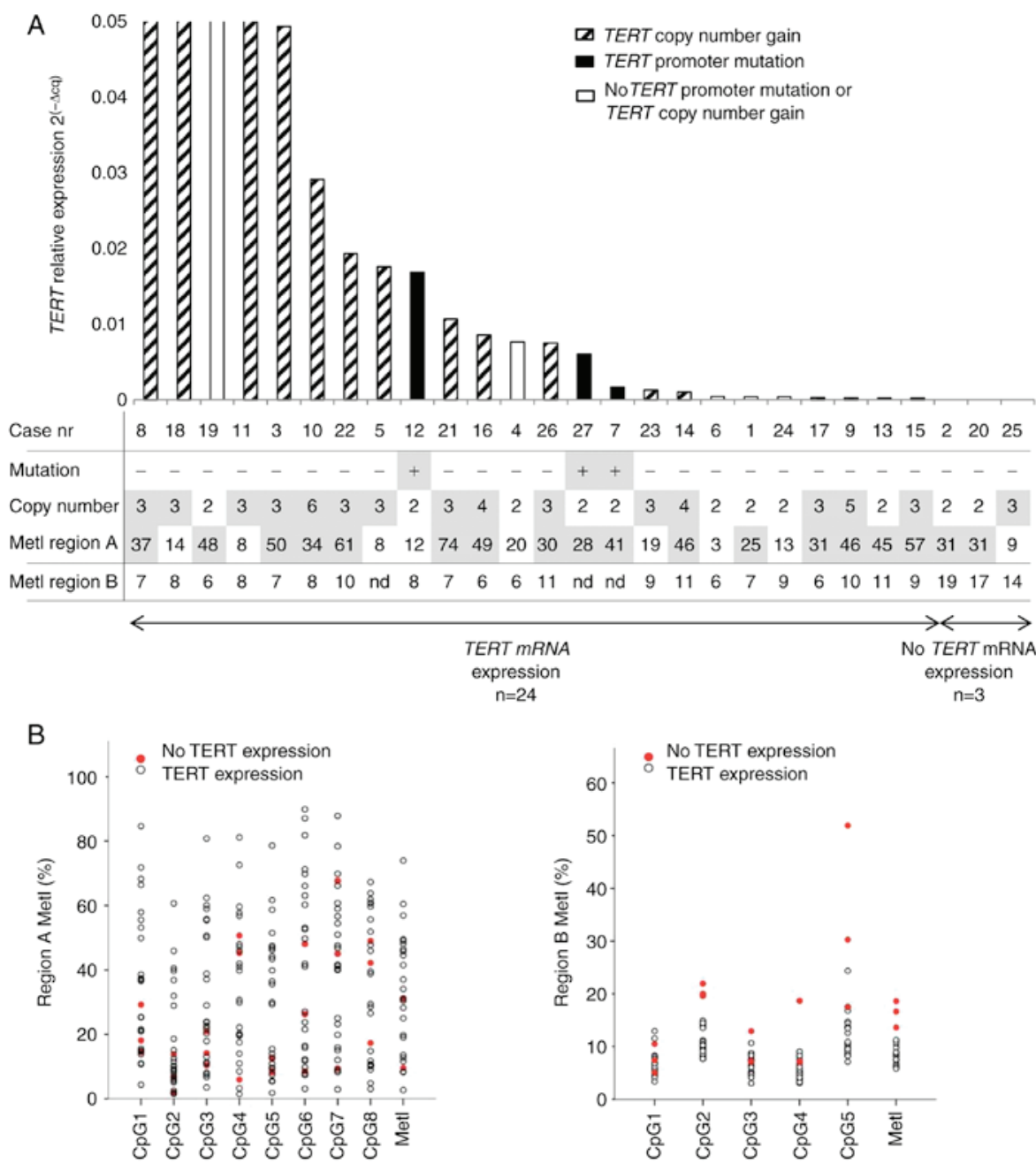

Figure 2. Results of expression of TERT, copy number gain, mutation status and promoter methylation in Regions A and B in 27 ACC cases. (A) Relative mRNA expression of TERT is shown at the top. Cases with a TERT promoter mutation are indicated by solid bars, cases with TERT copy number gain by striped bars, and cases without TERT promoter mutation or TERT copy number gain by unfilled bars. TERT mutations, copy numbers, and MetI (\%) for Regions A and B in the same ACC cases are shown below. Cases with mutation, copy number gain and increased MetI in Region A are highlighted (cut-off at $>20.4 \%$ ). (B) Scatterplots for 27 ACC samples with known TERT expression. Methylation densities are shown at specific CpG sites in addition to overall MetI in Region A and B. Tumors with and without TERT mRNA expression are indicated as red and open circles, respectively. TERT, telomerase reverse transcriptase; ACC, adrenocortical carcinoma; MetI, methylation index; nd, not determined. 
A

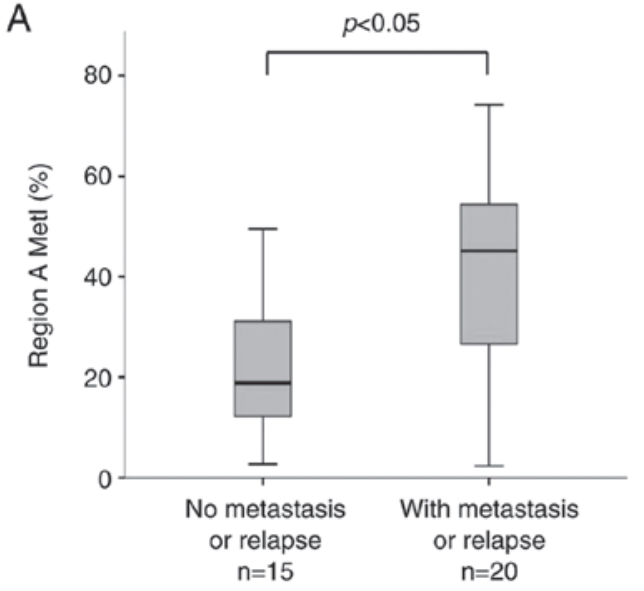

C

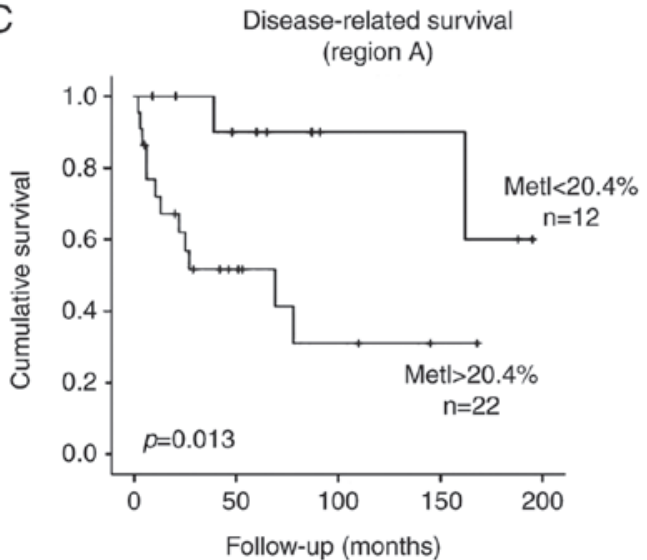

B

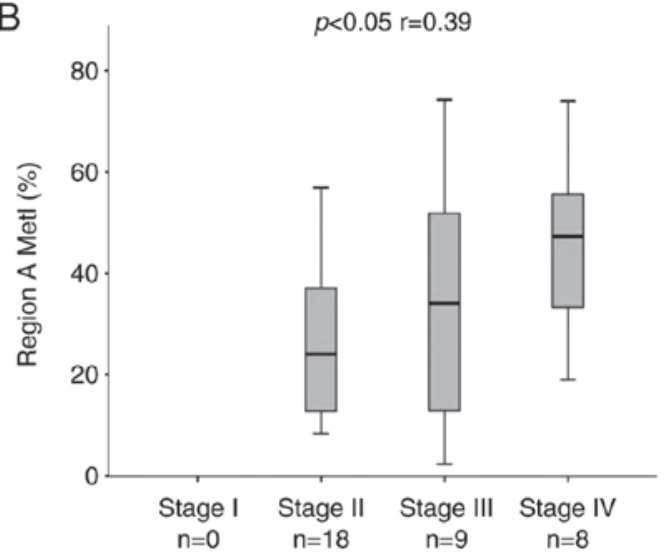

D

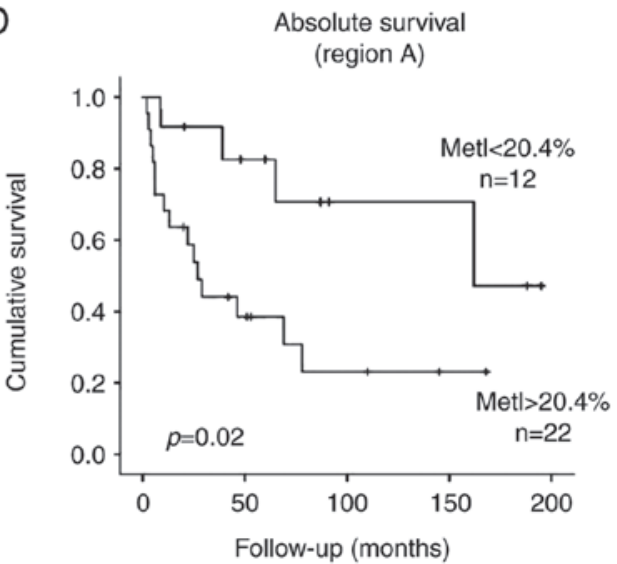

Figure 3. Boxplot presentation of TERT promoter methylation for Region A in relation to metastasis/recurrence and stage in ACC and Kaplan-Meier plots for survival analysis of ACC cases according to Region A TERT promoter methylation. (A) MetI in Region A according to the presence or lack of metastases or relapse in 35 ACC cases. (B) MetI in Region A according to Stage I, II, III and IV disease based on European Network for the Study of Adrenal Tumor score in 35 ACC cases. (C) Disease-related survival for 34 ACC cases. (D) Absolute survival for 34 ACC cases. Increased and decreased MetI in Region A refers to above and below the cut-off at 20.4\%, respectively. TERT, telomerase reverse transcriptase; ACC, adrenocortical carcinoma; MetI, methylation index; nd, not determined.

mechanism behind this telomerase activity, are known to occur in up to $10 \%$ of ACC cases and TERT expression is detected in the majority of tumors. Therefore, additional mechanisms are expected to be involved in the activation. Copy number gain and aberrant promoter methylation are additional frequently occurring alterations in this tumor entity. Genetic and epigenetic TERT alterations were present in the majority of ACC samples analyzed in the present study, supporting the hypothesis that the development of TERT-based diagnostic tools may be of value for improved diagnostics of this entity. Furthermore, increased methylation density in Region A was associated with metastatic disease, higher ENSAT stage and shorter survival rates, which may be further developed into an additional prognostic tool for ACC. Given the toxic effects of mitotane on the gastrointestinal system (anorexia, diarrhea, nausea, vomiting), the central nervous system (apathy, drowsiness, neuropathy, dizziness) and to the hormonal system (adrenal insufficiency) (6), a novel prognostic tool for ACC may be of important clinical significance for therapeutic decisions.

Gain of the TERT locus in chromosomal region 5p13.3 is a common event in ACC, which can be observed at the chromosome level (20) and for the individual gene locus (7-9). In the present study, increased TERT copy numbers were detected in 16/27 (59\%) of the ACC samples. These predominantly included gains, whereas amplifications were observed in a small number of tumors. These findings are in agreement with previous studies in which increased copy numbers were reported either in the form of gains, amplifications or both (7-9).

In the present study, TERT copy number gain or promoter mutations were present in $70 \%$ of ACC samples in a non-overlapping manner. The TERT mutation has previously been associated with induction of the expression of TERT (12). Copy number aberrations are characteristic of ACC but uncommon in adrenocortical adenomas (20). A copy number increase of the telomerase encoding regions has been suggested as a possible explanation for telomerase activation (21). This type of copy number gain has been found in several tumor types, and the components that build up the telomerase complex are located on chromosomal regions, which have been reported with frequent copy number increase (21). As TERT promoter mutations are known to confer increased transcription of $T E R T$, it is likely that $T E R T$ copy number gain or mutation may each contribute to the observed expression.

Epigenetic modifications, including DNA methylation of $\mathrm{CpG}$ sites, are major contributors to the construction and 
organization of the DNA molecule and also have an impact on gene expression. Altered $\mathrm{CpG}$ methylation has been shown in several cancer types and is a proposed therapeutic target (22), however, methylation densities in the TERT promoter of ACC have not been investigated despite lacking knowledge of the underlying cause for increased expression of TERT in these tumors. In the present study, high MetI in Region A was found to be associated with metastases and poorer survival rates in patients with ACC. Further evidence of this connection was shown by the association between MetI for Region A and ENSAT stage, where a higher ENSAT stage indicates more advanced cancer development. These findings are in line with previous observations in medullary thyroid carcinoma, where high MetI was associated with a shorter survival rate (13). Future investigations are required to investigate MetI in association with telomerase activity in ACC, which may provide additional information on the role of methylation levels in the TERT promoter.

A previous study (23) examined the methylation levels of $\mathrm{CpG}$ islands in ACC and found that genome-wide hypermethylation was associated with poor survival rate. However, the TERT locus was not investigated. The findings reported by Jouinot et al (23) suggest that TERT promoter hypermethylation at Region A may be due to a general hypermethylation phenotype. If so, TERT hypermethylation in Region A may be one of the important effects of such a profile due to its ability to influence telomere length and promote tumor cell survival. These results indicated the prognostic value of investigating methylation in this tumor type, which was further recognized in the results of the present study.

In the cohort of ACC cases used in the present study, the gene expression of TERT was found in $90 \%$ of the ACC cases. The majority of these tumors harbored TERT copy number gain, the C228T promoter mutation, or increased MetI in Region A, suggesting that these alterations contributed to the gene expression of TERT. No significant associations were revealed between the expression levels of TERT and copy numbers, mutation or MetI for Region A. This suggested that several different mechanisms are involved in the acquired increase of transcription, and that TERT copy number gain and increased TERT promoter methylation are two elements of the upregulation of TERT. Additional investigations are required to further understanding of the underlying mechanisms of the upregulation of TERT.

An inverse correlation was revealed between MetI for Region B and the expression of TERT. Low methylation in this region has been shown to be important for the transcription of TERT to occur in vitro by affecting the binding of the 11-zinc finger protein transcription factor CTCF (14). All ACC samples exhibiting TERT expression had low MetI at Region B. By contrast, the highest levels of Region B MetI were revealed in the three ACC samples in which the TERT gene was not expressed. Taken together, these observations supported the hypothesis that low methylation close to the transcription start site is a requisite for the transcription of TERT in the in vivo ACC tumor situation in addition to previous observations in vitro.

Telomere length and cancer development are considered to be associated, and telomere length in tumors and in constitutional tissues of cancer patients have been associated with cancer development and survival rates. For ACC, this has been supported by observations in Li-Fraumeni syndrome, in which the development of malignancies, including ACC, has been associated with shorter telomeres in TP53 mutation carriers (24). Induction of the expression of TERT is a major contributor to telomerase activation and subsequent elongation of telomeres. In line with this, the expression of TERT in ACC was positively correlated with telomere length, and telomere length was associated with patient survival rates. In the present study, mean relative telomere length was calculated using a qPCR-based method, which makes the results suitable for comparison only between tumors of this study. A sequencing-based method for calculating telomere length was used (8) for ACC, which found that the majority of ACC samples had shorter telomeres compared with matching normal samples. In the present study, a similar effect on telomere length was not observed, however the comparison was performed against independent NAT samples. In the same previous study, the results showed no association between telomere length and TERT mutation or TERT amplification, which is in accordance with the results of the present study (8).

Activation of a telomere-maintaining mechanism is a feature of ACC $(11,25)$, but has not been consistently found in adrenocortical adenomas or NAT (26). One mechanism that is expected to be operative in TERT-positive ACCs, is the activation of telomerase when the telomere length hits a critical low point. In the present study, $90 \%$ of ACC samples were positive for the expression of TERT, which is in agreement with a report of frequent telomerase activation in ACC based on a TRAP assay (11). Besides telomerase activation, homologous recombination has been described in elongation of telomeres in cancer, termed alternative lengthening of telomeres (ALT). Although telomerase activation is the common mechanism of telomere maintenance in ACC, a small number of ACC cases have been shown to utilize the ALT mechanisms or, rarely, both (11).

Although the present study did not demonstrate a significant association between TERT promoter methylation density, $T E R T$ copy number gain and expression of TERT, the increase in MetI and copy number was widely observed within these tumors, compared with NAT samples, and MetI in Region A was associated with poorer clinical outcome, which indicated that these alterations are of importance in this type of tumor. In conclusion, the present study provided evidence for epigenetic alterations of the TERT gene locus being of significant importance for the severity of ACC.

\section{Acknowledgements}

The authors would like to acknowledge Ms. Elisabeth Berg (Karolinska Institutet, Stockholm, Sweden) for her expert support in statistical analyses and Ms. Elisabet Ånfalk (Karolinska University Hospital, Stockholm, Sweden) for her assistance in tissue sample collection.

\section{Funding}

This study was supported by the Swedish Cancer Foundation (grant nos. 170613 and 170092), the Cancer Research Foundations of Radiumhemmet (grant nos. 174203 and 161143), 
Karolinska Institutet (Clinical Scientist Training Program Grant to Dr Fredrika Svahn), Stockholm County Council (grant no. Dnr LS 2016-1376) and the Swedish Society for Medical Research (to Dr C. Christofer Juhlin).

\section{Availability of data and materials}

The datasets used and/or analyzed during the current study are available from the corresponding author on reasonable request.

\section{Authors' contributions}

FS, AS, CCJ and CL conceived and designed the study. FS, JOP, OF, NW and NM researched and analysed data. TDM, $\mathrm{RK}, \mathrm{TC}, \mathrm{MB}$ and CCJ provided tissue samples and clinical details. FS, AS, CCJ and CL wrote, edited and reviewed the manuscript. All authors read and approved the final manuscript.

\section{Ethics approval and consent to participate}

All tissue samples were collected with informed consent and ethical permission granted from the Ethics Committee of Karolinska Institutet and from the International Review Board at Yale University.

\section{Patient consent for publication}

Not applicable.

\section{Competing interests}

The authors confirm that they have no competing interests.

\section{References}

1. MacNeil DE, Bensoussan HJ and Autexier C: Telomerase regulation from beginning to the end. Genes (Basel) 7: E64, 2016

2. Landa I, Ganly I, Chan TA, Mitsutake N, Matsuse M, Ibrahimpasic T, Ghossein RA and Fagin JA: Frequent somatic TERT promoter mutations in thyroid cancer: Higher prevalence in advanced forms of the disease. J Clin Endocrinol Metab 98: E1562-E1566, 2013.

3. Gisselsson D, Jonson T, Petersén A, Strombeck B, Dal Cin P, Höglund M, Mitelman F, Mertens F and Mandahl N: Telomere dysfunction triggers extensive DNA fragmentation and evolution of complex chromosome abnormalities in human malignant tumors. Proc Natl Acad Sci USA 98: 12683-12688, 2001.

4. Bilimoria KY, Shen WT, Elaraj D, Bentrem DJ, Winchester DJ, Kebebew E and Sturgeon C: Adrenocortical carcinoma in the United States: Treatment utilization and prognostic factors. Cancer 113: 3130-3136, 2008.

5. Payabyab EC, Balasubramaniam S, Edgerly M, Velarde M, Merino MJ, Venkatesan AM, Leuva H, Litman T, Bates SE and Fojo T: Adrenocortical cancer: A molecularly complex disease where surgery matters. Clin Cancer Res 22: 4989-5000, 2016.

6. Postlewait LM, Ethun CG, Tran TB, Prescott JD, Pawlik TM, Wang TS, Glenn J, Hatzaras I, Shenoy R, Phay JE, et al: Outcomes of adjuvant mitotane after resection of adrenocortical carcinoma: A 13 -institution study by the US adrenocortical carcinoma group. J Am Coll Surg 222: 480-490, 2016.

7. Juhlin CC, Goh G, Healy JM, Fonseca AL, Scholl UI, Stenman A, Kunstman JW, Brown TC, Overton JD, Mane SM, et al: Whole-exome sequencing characterizes the landscape of somatic mutations and copy number alterations in adrenocortical carcinoma. J Clin Endocrinol Metab 100: E493-E502, 2015.
8. Zheng S, Cherniack AD, Dewal N, Moffitt RA, Danilova L, Murray BA, Lerario AM, Else T, Knijnenburg TA, Ciriello G, et al: Comprehensive pan-genomic characterization of adrenocortical carcinoma. Cancer Cell 29: 723-736, 2016.

9. Assie G, Letouzé E, Fassnacht M,Jouinot A, Luscap W, Barreau O, Omeiri H, Rodriguez S, Perlemoine K, Rene-Corail F, et al: Integrated genomic characterization of adrenocortical carcinoma. Nat Genet 46: 607-612, 2014.

10. Liu T, Brown TC, Juhlin CC, Andreasson A, Wang $\mathrm{N}$, Bäckdahl M, Healy JM, Prasad ML, Korah R, Carling T, et al: The activating TERT promoter mutation C228T is recurrent in subsets of adrenal tumors. Endocr Relat Cancer 21: 427-434, 2014.

11. Else T, Giordano TJ and Hammer GD: Evaluation of telomere length maintenance mechanisms in adrenocortical carcinoma. J Clin Endocrinol Metab 93: 1442-1449, 2008.

12. Horn S, Figl A, Rachakonda PS, Fischer C, Sucker A, Gast A, Kadel S, Moll I, Nagore E, Hemminki K, et al: TERT promoter mutations in familial and sporadic melanoma. Science 339: 959-961, 2013.

13. Wang N, Kjellin H, Sofiadis A, Fotouhi O, Juhlin CC, Bäckdahl M, Zedenius J, Xu D, Lehtiö J and Larsson C: Genetic and epigenetic background and protein expression profiles in relation to telomerase activation in medullary thyroid carcinoma. Oncotarget 7: 21332-21346, 2016.

14. Renaud S, Loukinov D, Abdullaev Z, Guilleret I, Bosman FT, Lobanenkov V and Benhattar J: Dual role of DNA methylation inside and outside of CTCF-binding regions in the transcriptional regulation of the telomerase hTERT gene. Nucleic Acids Res 35: 1245-1256, 2007.

15. DeLellis RA, Lloyd RV, Heitz PU and Eng C: Pathology and genetics of tumours of endocrine organs. Third edition. WHO Classification of Tumours, Volume 8, 2004.

16. Moran B, Das S, Smeets D, Peutman G, Klinger R, Fender B, Connor K, Ebert M, Gaiser T, Prehn JH, et al: Assessment of concordance between fresh-frozen and formalin-fixed paraffin embedded tumor DNA methylation using a targeted sequencing approach. Oncotarget 8: 48126-48137, 2017.

17. Wang N, Xu D, Sofiadis A, Höög A, Vukojević V, Bäckdahl M, Zedenius $J$ and Larsson C: Telomerase-dependent and independent telomere maintenance and its clinical implications in medullary thyroid carcinoma. J Clin Endocrinol Metab 99: E1571-E1579, 2014.

18. Cawthon RM: Telomere measurement by quantitative PCR. Nucleic Acids Res 30: e47, 2002

19. Mannelli M, Gelmini S, Arnaldi G, Becherini L, Bemporad D, Crescioli C, Pazzagli M, Mantero F, Serio M and Orlando C: Telomerase activity is significantly enhanced in malignant adrenocortical tumors in comparison to benign adrenocortical adenomas. J Clin Endocrinol Metab 85: 468-470, 2000.

20. Kjellman M, Kallioniemi OP, Karhu R, Höög A, Farnebo LO, Auer G, Larsson C and Bäckdahl M: Genetic aberrations in adrenocortical tumors detected using comparative genomic hybridization correlate with tumor size and malignancy. Cancer Res 56: 4219-4223, 1996

21. Cao Y, Bryan TM and Reddel RR: Increased copy number of the TERT and TERC telomerase subunit genes in cancer cells. Cancer Sci 99: 1092-1099, 2008.

22. Hamidi T, Singh AK and Chen T: Genetic alterations of DNA methylation machinery in human diseases. Epigenomics 7: 247-265, 2015.

23. Jouinot A, Assie G, Libe R, Fassnacht M, Papathomas T, Barreau O, de la Villeon B, Faillot S, Hamzaoui N, Neou M, et al: DNA Methylation is an independent prognostic marker of survival in adrenocortical cancer. J Clin Endocrinol Metab 102: 923-932, 2017.

24. Tabori U, Nanda S, Druker H, Lees J and Malkin D: Younger age of cancer initiation is associated with shorter telomere length in Li-Fraumeni syndrome. Cancer Res 67: 1415-1418, 2007.

25. Hirano Y, Fujita K, Suzuki K, Ushiyama T, Ohtawara Y and Tsuda F: Telomerase activity as an indicator of potentially malignant adrenal tumors. Cancer 83: 772-776, 1998.

26. Else T: Telomeres and telomerase in adrenocortical tissue maintenance, carcinogenesis, and aging. J Mol Endocrinol 43: 131-141, 2009. 